\title{
Silencing LncRNA SCAMP1 inhibits cell proliferation, induces G0/G1 arrest and apoptosis in hepatocellular carcinoma
}

Xiaochen Ma

Xiangyang Sun ( $\nabla$ xiang_yangsun98@163.com )

Caixia Li

Kai Zhang

Yang Xie

\section{Research Article}

Keywords: hepatocellular carcinoma, SCAMP1, proliferation, apoptosis

Posted Date: October 15th, 2021

DOl: https://doi.org/10.21203/rs.3.rs-956337/v1

License: (c) (i) This work is licensed under a Creative Commons Attribution 4.0 International License.

Read Full License 


\section{Abstract}

Background: Long noncoding RNA secretory carrier membrane protein 1 (SCAMP1) has been recently reported to be an oncogene in glioma and ovarian cancer. However, little is known about the clinical significance and functional role of SCAMP1 in hepatocellular carcinoma (HCC).

Methods: The expression of SCAMP1 was determined in tissue samples and cell lines using quantitative reverse transcription PCR (qRT-PCR). The clinical significance of SCAMP1 in HCC was evaluated using chi-squared test, Kaplan-Meier survival, as well as univariate and multivariate analysis. Cell proliferation, cell cycle distribution and apoptosis were evaluated using CCK-8 assay, colony formation assay, Flow cytometry analysis. Related protein expression levels were measured by western blot analysis.

Results: We found SCAMP1 expression levels were remarkably up-regulated in HCC tissues compared to that in matched adjacent tissues. Increased SCAMP1 expression was significantly correlated with large tumor size, advanced TNM stage and poor survival prognosis in HCC. Knockdown of SCAMP1 significantly inhibited cell proliferation, induced G0/G1 phase arrest and apoptosis in HepG2 and SNU182 cells. More importantly, knockdown of SCAMP1 downregulated the protein levels of CDK4, Cyclin D1 and Bcl-2, while upregulated the protein levels of p21, p53 and Bax.

Conclusions: In summary, our study clarified a positive correlation of the SCAMP1 levels with the HCC cell proliferation.

\section{Background}

Hepatocellular carcinoma (HCC), accounting for almost $80 \%$ of all primary liver cancers, is clinically characterized by high prevalence, high mortality rate and drug resistance $(1,2)$. At present, significant advances have been made in surgical resection, chemotherapy or radiotherapy for HCC, but the five-year survival rate is generally below $50 \%$ for lacking of specific indicators in the early stage (3-5). Therefore, identification of new therapeutic targets is of great importance for developing effective therapeutic strategy for HCC.

It has been reported that aberrantly expressed long non-coding RNAs (IncRNAs) were aberrantly expression in the occurrence and development of HCC, which are identified as the oncogenes or tumor suppressors. For example, SAMMSON expression level was upregulated in HCC tissues, and patients with high SAMMSON level had significantly lower overall rate within five years after admission (6).

Upregulation of PDPK2P was clinically associated with a larger tumor embolus, low differentiation, and poor survival, as well as functionally promoted the cell proliferation and metastasis in HCC (7). On the contrary, LINC01554 was downregulated in HCC, and its expression was correlated with tumor recurrence, advanced TNM stage and reduced long-term survival rate of patients (8). In addition, RUNX1-IT1 (9), EPB41L4A-AS2 (10) and TPTEP1 (11) exert tumor suppressive properties by inhibiting HCC cell growth and proliferation. 
Recently, a IncRNA transcript secretory carrier membrane protein 1 (SCAMP1) functions as a carrier and participates in post-Golgi recycling pathway (12), which has been reported to be dysregulated and correlated with the development of various tumors $(13,14)$. For instance, Wang et al $(15)$ identified that SCAMP1, as one of the key IncRNAs, is a prognostic biomarker of pancreatic cancer by performing bioinformatic analysis. Functionally, inhibition of SCAMP1 significantly restrained the cell proliferation, migration and invasion, as well as promoted apoptosis by acting as a molecular sponge of miR-499a$5 p$ (16). SCAMP1 depletion attenuated cell viability and promoted apoptosis in pediatric renal cell carcinoma under oxidative stress (17). Similarly, Song et al (18) found that SCAMP1 was highly expressed in ovarian cancer and targets miR-137/CXCL12 axis to boost cell invasion and angiogenesis. Based on these evidences, we thus speculated that SCAMP1 might be an oncogene in the development of HCC. To validate our hypothesis, this study was performed to investigate the expression levels, clinical significance, biological effects and related molecular mechanisms of SCAMP1 in HCC.

\section{Materials And Methods}

\section{Clinical tissue samples}

Tumor tissues and matched adjacent non-cancer tissues (at least $5 \mathrm{~cm}$ away from the edge of tumor) were collected from 76 cases of HCC patients who received hepatectomy at Qilu Hospital of Shandong University (Shandong, China) between January 2015 and December 2019 with informed consent from all patients. Before surgery, none of HCC patients received any chemotherapy, radiation therapy or immunotherapy and targeted therapy. The basic demographic and clinicopathological features for HCC patients were described in Table 1. All tissue samples were immediately frozen in liquid nitrogen and stored at $-80^{\circ} \mathrm{C}$ for further analysis. This study was approved by Institutional Ethics Committee of Qilu Hospital of Shandong University (Approval no. QS-A87D1, Shandong, China) based on the Declaration of Helsinki.

\section{Cell culture and transfection}

A normal transformed human liver epithelial-3 cell line (THLE-3) and two HCC cell lines (HepG2 and SNU182) were provided by American Type Culture Collection (ATCC, Manassas, VA, USA). These cell lines were cultured in Dulbecco's Modified Eagle's Medium (DMEM, Gibco, Grand Island, NY, USA) with 10\% fetal bovine serum (FBS, Gibco) and maintained at $37^{\circ} \mathrm{C}$ in a humidified atmosphere of $5 \% \mathrm{CO}_{2}$. For cell transfection, two small interfering RNA targeting SCAMP1 (si-SCAMP1\#1 and si-SCAMP1\#2) and negative control (si-NC) synthesized by GenePharma (Shanghai, China) were transfected into HepG2 and SNU-182 cells using Lipofectamine 2000 reagent (Invitrogen, California, USA).

Quantitative reverse transcription PCR ( $q R T-P C R)$

Total RNA was isolated from tissue samples or cell lines with TRIzol reagent (Life technologies, Carlsbad, CA, USA) and reverse transcription was performed using Prime Script RT reagent Kit (Takara Bio, Japan) following the manufactures' instructions. Quantitative reverse transcription PCR was carried out using 
SYBR Green qPCR Master Mix (Takara Bio, Japan) on ABI Prism 7500 Fast Real-Time PCR system (Applied Biosystems, USA) with the primer sequences as follows: SCAMP1 forward: 5'-

TCCCTTCAAGCCTCCACCA-3' and reverse: 5'-GACTGAGGTTTTGCATTTCTCGTTC-3'; GAPDH forward: 5'GGTGAAGGTCGGAGTCAACG-3' and 5'-GCATCGCCCCACTTGATTTT-3'. Relative expression level of SCAMP1 was normalized to that of endogenous control (GAPDH) using the $2-\Delta \Delta \mathrm{Ct}$ method. Three independent experiments were conducted.

\section{CCK-8 assay}

After $48 \mathrm{~h}$ transfection, cells were seeded into 96 -well plates at a density of $3 \times 10^{3}$ cells per well and cultured overnight. At $0,24,48$ and $72 \mathrm{~h}, 10 \mu \mathrm{L}$ of CCK-8 (Cell counting Kit-8) solution (Dojindo, Kumamoto, Japan) was added to cells in each well. After another $2 \mathrm{~h}$ incubation at $37^{\circ} \mathrm{C}$, the absorbance was measured at $450 \mathrm{~nm}$ by a microplate reader. Three independent experiments were conducted.

\section{Colony formation assay}

After $48 \mathrm{~h}$ transfection, cells at a density of 500 cells per well were seeded into six-well plates and cultured for consecutive two weeks to form cell colony. Subsequently, cell colonies were washed twice with PBS and fixed with $4 \%$ paraformaldehyde for $30 \mathrm{~min}$. After stained with crystal violet for $20 \mathrm{~min}$, the colonies were photographed and counted under a light microscope.

\section{Flow cytometry assay}

For cell cycle analysis, transfected cells were harvested and fixed with $70 \%$ ethanol overnight at $4{ }^{\circ} \mathrm{C}$. After washed with phosphate buffer saline (PBS), cells were incubated with $0.5 \mathrm{~mL}$ of propidium iodide (PI)/RNase Staining Buffer (BD biosciences, San Jose, CA, USA) for 20 min at room temperature. Cell cycle distribution was analyzed by a FACSCalibur flow cytometer (BD Biosciences). For the analysis of apoptosis, transfected cells were washed with PBS twice and stained with Annexin V-FITC and PI for 15 min at room temperature in darkness. The percentage of early (Annexin $\mathrm{V}+/ \mathrm{PI}$ ) and late (Annexin $\mathrm{V}+/ \mathrm{PI}+$ ) apoptosis was quantified using flow cytometry (BD Biosciences).

\section{Western blot analysis}

Total protein samples were extracted with radioimmunoprecipitation assay (RIPA) lysis buffer (Beyotime, Shanghai, China) and the lysates were incubated for $30 \mathrm{~min}$ on ice and centrifugated at $300 \mathrm{x} \mathrm{g}$ for 10 min at $4{ }^{\circ} \mathrm{C}$. After quantified using a bicinchoninic acid (BCA) protein assay kit (Beyotime), equal amount of protein samples was separated on $10 \%$ sodium dodecyl sulphate (SDS)-polyacrylamide gel electrophoresis and transferred onto polyvinylidene fluoride (PVDF) membranes (Millipore, Billerica, MA, USA). The membranes were blocked with $5 \%$ non-fat milk and then incubated with primary antibodies against CDK4, Cyclin D1, p21, p53, Bcl-2, Bax and GAPDH (all from Abcam, Cambridge, MA, USA) overnight at $4{ }^{\circ} \mathrm{C}$. After washed with PBS twice, the membranes were incubated with horseradish peroxidase-conjugated secondary antibodies (Cell Signaling Technology) for $2 \mathrm{~h}$ at room temperature. 
The protein bands were visualized using enhanced chemiluminescence (ECL) reagent (Millipore) and analyzed using Amersham ${ }^{\mathrm{TM}}$ Imager 680 from GE Healthcare Life Sciences with glyceraldehyde-3phosphate dehydrogenase (GAPDH) as the internal control.

\section{Statistical analysis}

Experimental data were analyzed by GraphPad Prism 6.0 Software (GraphPad Inc., San Diego, CA, USA) and expressed as mean \pm standard deviation (SD) from three biological replicates of each experiment. The associations between SCAMP1 expression and clinicopathological features were studied using chisquare test. Overall survival curves were plotted based on Kaplan-Meier method and estimated by the logrank test. Univariate and multivariate Cox regression analyses were used to determine the independent factors that influenced survival. Paired $t$ test was used to analyze differences between two types of tissues. One-way ANOVA and Tukey test were used to analyze differences among different cell transfection groups. The values of all $p$ less than 0.05 were considered to be statistically significant level.

\section{Results}

SCAMP1 was upregulated in HCC tissues and cell lines

Using qRT-PCR, we determined the expression level of SCAMP1 in tumor tissues and adjacent non-cancer tissues derived from 76 cases of HCC patients. As shown in Figure 1A, SCAMP1 expression level was significantly upregulated in HCC tissues compared with adjacent tissues. In addition, we detected the expression level of SCAMP1 in HCC cell lines. Consistently, elevated expression of SCAMP1 was also observed in two HCC cell lines (HepG2 and SNU-182), in comparison with human liver epithelial-3 cell line THLE-3 (Figure 1B).

High level of SCAMP1 was associated with poor prognosis of HCC patients

Based on the expression level of SCAMP1 in HCC tissues, we next analyzed the clinical significance of SCAMP1 in HCC. At first, 76 cases of HCC patients were divided into two subgroups (high/low SCAMP1 level) using the median of the cohort as a cut-off value. The correlation analysis between SCAMP1 expression and clinicopathological features of HCC patients was displayed in Table 1, which indicated that high expression of SCAMP1 was positively correlated with large tumor size $(p=0.003)$ and advanced TNM stage $(p=0.006)$. Through survival analysis, it was found that patients with the high expression of SCAMP1 had a shorter overall survival time than those with low SCAMP1 expression (Figure 2, $p=$ 0.0168). Furthermore, the results from Cox regression analysis (Table 2 ) demonstrated that SCAMP1 expression was an independent prognostic factor for HCC patient survival (HR $=1.006, p=0.018)$, in addition to tumor size $(\mathrm{HR}=2.015, p=0.026)$.

SCAMP1 knockdown suppressed cell proliferation, induced GO/G1 phase arrest and apoptosis in HCC cells 
The increased expression of SCAMP1 correlated with large tumor size led us to hypothesize that SCAMP1 might promote the HCC cell proliferation in vitro. To this end, SCAMP1 expression was stably depleted in HepG1 and SNU-182 cells with different specific siRNAs. The knockdown efficiency was verified using qRT-PCR (Figure 3A). The results of CCK-8 assay showed that SCAMP1 knockdown significantly inhibited the proliferation of HepG1 (Figure 3B) and SNU-182 (Figure 3C) cells. We observed that si-SCAMP1\#1 transfection showed stronger suppressive effects on SCAMP1 expression and cell growth and thus selected it for the subsequent experiments. Next, we further confirmed the suppressive effects of SCAMP1 knockdown on cell proliferation by the results of colony formation assay (Figure 3D). Considering the close relationship between cell proliferation and cell cycle progression/apoptosis status, we next performed flow cytometry analysis to investigate the effects of SCAMP1 knockdown on HCC cell cycle distribution and apoptosis. As depicted in Figure 4A, the percentage of cells at G0/G1 phase was significantly increased, while cells at $\mathrm{S}$ and $\mathrm{G} 2 / \mathrm{M}$ phase were accordingly decreased in si-SCAMP1\#1 transfection group compared with si-NC group in both HepG2 and SNU-182 cells. Similarly, SCAMP1 knockdown remarkedly promoted cell apoptosis, including early and late apoptosis in both HepG2 and SNU-182 cells (Figure 4B).

\section{SCAMP1 knockdown activated p53 signaling pathway in HCC cells}

To clarify the molecular mechanisms underlying SCAMP1 knockdown regulating HCC cell proliferation, G1/S transition and apoptosis, we analyzed the expression of CDK4/Cyclin D1, associated with G1/S transition and p53 target genes, including p21, p53, Bcl-2 and Bax using western blot analysis. Our results showed that knockdown of SCAMP1 obviously downregulated the expression levels of CDK4, Cyclin D1 and anti-apoptotic Bcl-2, while upregulated the expression levels of p21, p53 and pro-apoptotic Bax in both HepG2 (Figure 5A) and SNU-182 (Figure 5B) cells.

\section{Discussion}

This study focused on the expression level and role of SCAMP1 in HCC. We first showed that SCAMP1 was highly expressed in HCC tissues and cell lines, and elevated SCAMP1 expression was correlated with tumor size, TNM stage and poor prognosis in HCC patients. Consistent with the upregulation of SCAMP1 in HCC, several studies have reported that the expression levels of SCAMP1 were increased in ovarian cancer (18), glioma (16) and pediatric renal cell carcinoma (17). At clinical level, Song et al (18) utilized TCGA database to observe that ovarian cancer patients with high SCAMP1 level had poorer overall survival rates than those with low SCAMP1. The expression level of SCAMP1 was positively correlated with the pathological grades of glioma and higher SCAMP1 expression indicated poorer overall survival (16). These results indicated that SCAMP1 might be a potential biomarker in clinical application.

Our in vitro experiments showed that SCAMP1 knockdown suppressed cell proliferation, induced cell cycle G0/G1 phase arrest and promoted apoptosis in two HCC cell lines (HepG2 and SNU-182), which suggested that SCAMP1 can be used as an oncogene to promote HCC cell proliferation. In fact, SCAMP1 has been reported to play oncogenic role in other tumors. For instance, inhibition of SCAMP1 significantly 
restrained the cell proliferation, migration and invasion, as well as promoted apoptosis in glioma (16). SCAMP1 depletion attenuated cell viability and promoted apoptosis under $\mathrm{H}_{2} \mathrm{O}_{2}$ treatment in pediatric renal cell carcinoma (17). Downregulation of SCAMP1 suppressed cell proliferation, migration and invasion in pancreatic and gallbladder cancer (19).

In terms of molecular mechanism, our data further demonstrated that SCAMP1 knockdown reduced the expression of CDK4, Cyclin D1 and Bcl-2, but promoted the expression of p21, p53 and Bax in both HepG2 and SNU-182 cells. It has been widely accepted that deregulated growth is a unique characteristic of cancer cells and a primary requirement for processes in cancer progression (20). And there is a strong link between cell cycle deregulation and carcinogenesis (21). Cell proliferation is primarily regulated by the cell cycle regulation, including four distinct sequential phases (G0/G1, S and $\mathrm{G} 2 / \mathrm{M})$ to monitor DNA integrity $(22,23)$. In eukaryotes, cell cycle is regulated by cyclin-dependent kinases (CDKs), Cyclins and CDK inhibitors (CDKIs) (24). By flow cytometry analysis, we found that the inhibitory effect of SCAMP1 knockdown on HCC cell proliferation was associated with the blockage of G1 to S progression. As one of the main checkpoints of cell cycle, G1/S transition is responsible for initiation and completion of DNA replication, which is strongly regulated by the combined activity of CDK4/Cyclin D1 and p21 $(25,26)$. Here, SCAMP1 knockdown induced cell cycle G0/G1 phase arrest, which might be correlated with decreased CDK4/Cyclin D1 and increased p21 expression induced by depletion of SCAMP1. P53, the tumor suppressor gene product, can directly regulate the p21 gene, which encodes a universal inhibitor of CDKs, to inhibit the cell cycle progression (27). Apoptosis is a process of programmed cell death and caspase-cascade is a central part of cell apoptosis, which is regulated by various kinds of molecules, including Bcl-2 family proteins (28). Bcl-2 family plays a pivotal role in either inhibiting Bcl-2 or promoting Bax cell death $(29,30)$. It has been reported that Bax inactivating $\mathrm{Bcl}-2$ proteins regulates the apoptosis mediated by mitochondria and the ratio of $\mathrm{Bax}$ to $\mathrm{Bcl}-2$ proteins increases during apoptosis induction (31). Moreover, $\mathrm{Bcl}-2$ and $\mathrm{Bax}$ are the downstream target genes of p53. As our best knowledge, the tumor suppressor protein p53 has a critical role in regulation of the Bcl-2 family, including antiapoptotic protein $\mathrm{Bcl}-2$ and proapoptotic protein $\mathrm{Bax}(32,33)$. Thus, our findings revealed that SCAMP1 knockdown inhibited HCC cell proliferation via inducing cell cycle G0/G1 arrest and apoptosis. Of course, there were some limitations and future research prospects in our next work, including SCAMP1 overexpression experiments, the regulatory mechanism of SCAMP1 on cell invasion and metastasis, and identification on the potential effectors (such as microRNA) of SCAMP1.

\section{Conclusions}

In conclusion, our work demonstrated for the first time that SCAMP1 was overexpressed in HCC tissues and predicted poor prognosis. Moreover, SCAMP1 knockdown negatively regulated HCC cell growth and proliferation, which might be associated with the activation of p53 signaling pathway. Our data provide new insight into the oncogenic roles of SCAMP1 in HCC progression, which may become a potential therapeutic target for HCC. 


\section{Declarations}

\section{Ethical approval and consent to participate}

Written informed consent was obtained from all participants and the work was accepted by the Institutional Ethics Committee of Qilu Hospital of Shandong University (Approval no. QS-A87D1, Shandong, China) based on the Declaration of Helsinki.

\section{Authors' contributions}

SXY designed this research. MXC carried out most experiments in this work and drafted this manuscript. LCX performed statistical analysis. ZK and XY help to draft the manuscript. All authors read and approved the final manuscript.

\section{Availability of data and materials}

All data generated or analyzed during this study are included in this published article.

\section{Consent for publication}

Not applicable.

\section{Funding}

This study was approved by the China Health Promotion Foundation (ChiCTR2100042773).

\section{Conflict of interest}

The authors declare that they have no conflict of interest.

\section{Abbreviations}

HCC, hepatocellular carcinoma; SCAMP1, secretory carrier membrane protein 1; qRT-PCR, quantitative reverse transcription PCR; ATCC, American Type Culture Collection; DMEM, Dulbecco's modified Eagle's medium; FBS, fetal bovine serum; PBS, phosphate buffer saline; 3'-UTRs, 3'-untranslated regions; siRNAs, small interfering RNAs; RIPA, radioimmunoprecipitation assay; PVDF, polyvinylidene fluoride; CCK-8, Cell Counting Kit-8

\section{References}

1. Craig AJ, von Felden J, Garcia-Lezana T, Sarcognato S, Villanueva A. Tumour evolution in hepatocellular carcinoma. Nature reviews Gastroenterology \& hepatology. 2020 Mar;17(3):139-52. PubMed PMID: 31792430. 
2. Yang JD, Hainaut P, Gores GJ, Amadou A, Plymoth A, Roberts LR. A global view of hepatocellular carcinoma: trends, risk, prevention and management. Nature reviews Gastroenterology \& hepatology. 2019 Oct;16(10):589-604. PubMed PMID: 31439937. Pubmed Central PMCID: 6813818.

3. Saillard C, Schmauch B, Laifa O, Moarii M, Toldo S, Zaslavskiy M, et al. Predicting survival after hepatocellular carcinoma resection using deep-learning on histological slides. Hepatology. 2020 Feb 28. PubMed PMID: 32108950.

4. Imamura H, Matsuyama Y, Tanaka E, Ohkubo T, Hasegawa K, Miyagawa S, et al. Risk factors contributing to early and late phase intrahepatic recurrence of hepatocellular carcinoma after hepatectomy. Journal of hepatology. 2003 Feb;38(2):200-7. PubMed PMID: 12547409.

5. Liu PH, Hsu CY, Hsia CY, Lee YH, Su CW, Huang YH, et al. Prognosis of hepatocellular carcinoma: Assessment of eleven staging systems. Journal of hepatology. 2016 Mar;64(3):601-8. PubMed PMID: 26551516.

6. Yang S, Cai H, Hu B, Tu J. LncRNA SAMMSON negatively regulates miR-9-3p in hepatocellular carcinoma cells and has prognostic values. Bioence Reports. 2019;39(7).

7. Pan W, Li W, Zhao J, Huang Z, Zhao J, Chen S, et al. IncRNA-PDPK2P promotes hepatocellular carcinoma progression through the PDK1/AKT/Caspase 3 pathway. Molecular oncology. 2019 Oct;13(10):2246-58. PubMed PMID: 31368655. Pubmed Central PMCID: PMC6763783. Epub 2019/08/02. eng.

8. Ding Y, Sun Z, Zhang S, Chen Y, Zhou B, Li G, et al. Down-regulation of Long Non-coding RNA LINC01554 in Hepatocellular Cancer and its Clinical Significance. Journal of Cancer. 2020;11(11):336974. PubMed PMID: 32231743. Pubmed Central PMCID: 7097951.

9. Yan PH, Wang L, Chen H, Yu FQ, Guo L, Liu Y, et al. LncRNA RUNX1-IT1 inhibits proliferation and promotes apoptosis of hepatocellular carcinoma by regulating MAPK pathways. European review for medical and pharmacological sciences. 2019 Oct;23(19):8287-94. PubMed PMID: 31646558. Epub 2019/10/28. eng.

10. Wang YG, Wang T, Shi M, Zhai B. Long noncoding RNA EPB41L4A-AS2 inhibits hepatocellular carcinoma development by sponging miR-301a-5p and targeting FOXL1. Journal of experimental \& clinical cancer research : CR. 2019 Apr 10;38(1):153. PubMed PMID: 30971290. Pubmed Central PMCID: PMC6458726. Epub 2019/04/12. eng.

11. Ding H, Liu J, Zou R, Cheng P, Su Y. Long non-coding RNA TPTEP1 inhibits hepatocellular carcinoma progression by suppressing STAT3 phosphorylation. Journal of experimental \& clinical cancer research : CR. 2019 May 9;38(1):189. PubMed PMID: 31072375. Pubmed Central PMCID: PMC6509822. Epub 2019/05/11. eng. 
12.

Liao H, Zhang J, Shestopal S, Szabo G, Castle A, Castle D. Nonredundant function of secretory carrier membrane protein isoforms in dense core vesicle exocytosis. American journal of physiology Cell physiology. 2008 Mar;294(3):C797-809. PubMed PMID: 18171723.

13. Vadakekolathu J, Al-Juboori SIK, Johnson C, Schneider A, Buczek ME, Di Biase A, et al. MTSS1 and SCAMP1 cooperate to prevent invasion in breast cancer. Cell death \& disease. 2018 Mar 1;9(3):344. PubMed PMID: 29497041. Pubmed Central PMCID: 5832821.

14. Biewenga P, Buist MR, Moerland PD, Ver Loren van Themaat E, van Kampen AH, ten Kate FJ, et al. Gene expression in early stage cervical cancer. Gynecologic oncology. 2008 Mar;108(3):520-6. PubMed PMID: 18191186.

15. Wang W, Lou W, Ding B, Yang B, Lu H, Kong Q, et al. A novel mRNA-miRNA-IncRNA competing endogenous RNA triple sub-network associated with prognosis of pancreatic cancer. Aging. 2019 May 6;11(9):2610-27. PubMed PMID: 31061236. Pubmed Central PMCID: 6535056.

16. Zong Z, Song Y, Xue Y, Ruan X, Liu X, Yang C, et al. Knockdown of LncRNA SCAMP1 suppressed malignant biological behaviours of glioma cells via modulating miR-499a-5p/LMX1A/NLRC5 pathway. Journal of cellular and molecular medicine. 2019 Aug;23(8):5048-62. PubMed PMID: 31207033. Pubmed Central PMCID: PMC6653555. Epub 2019/06/18. eng.

17. Shao Q, Wang Q, Wang J. LncRNA SCAMP1 regulates ZEB1/JUN and autophagy to promote pediatric renal cell carcinoma under oxidative stress via miR-429. Biomedicine $\&$ pharmacotherapy $=$ Biomedecine \& pharmacotherapie. 2019 Dec;120:109460. PubMed PMID: 31550675. Epub 2019/09/25. eng.

18. Song R, Liu Z, Lu L, Liu F, Zhang B. Long Noncoding RNA SCAMP1 Targets miR-137/CXCL12 Axis to Boost Cell Invasion and Angiogenesis in Ovarian Cancer. DNA and cell biology. 2020 Jun;39(6):1041-50. PubMed PMID: 32401536. Epub 2020/05/14. eng.

19. Yang S, Lee KT, Lee JY, Lee JK, Lee KH, Rhee JC. Inhibition of SCAMP1 suppresses cell migration and invasion in human pancreatic and gallbladder cancer cells. Tumour biology : the journal of the International Society for Oncodevelopmental Biology and Medicine. 2013 Oct;34(5):2731-9. PubMed PMID: 23653380. Epub 2013/05/09. eng.

20. Vermeulen $\mathrm{K}$, Van Bockstaele DR, Berneman ZN. The cell cycle: a review of regulation, deregulation and therapeutic targets in cancer. Cell proliferation. 2003 Jun;36(3):131-49. PubMed PMID: 12814430. Pubmed Central PMCID: 6496723.

21. Nguyen-Ba G, Vasseur P. Epigenetic events during the process of cell transformation induced by carcinogens (review). Oncology reports. 1999 Jul-Aug;6(4):925-32. PubMed PMID: 10373683. 
22. Brooks G, La Thangue NB. The cell cycle and drug discovery: the promise and the hope. Drug discovery today. 1999 Oct;4(10):455-64. PubMed PMID: 10481141.

23. Suryadinata R, Sadowski M, Sarcevic B. Control of cell cycle progression by phosphorylation of cyclin-dependent kinase (CDK) substrates. Bioscience reports. 2010 Mar 17;30(4):243-55. PubMed PMID: 20337599.

24. Li JP, Yang YX, Liu QL, Pan ST, He ZX, Zhang X, et al. The investigational Aurora kinase A inhibitor alisertib (MLN8237) induces cell cycle G2/M arrest, apoptosis, and autophagy via p38 MAPK and Akt/mTOR signaling pathways in human breast cancer cells. Drug design, development and therapy. 2015;9:1627-52. PubMed PMID: 25834401. Pubmed Central PMCID: 4365748.

25. Schwartz GK, Shah MA. Targeting the cell cycle: a new approach to cancer therapy. Journal of clinical oncology : official journal of the American Society of Clinical Oncology. 2005 Dec 20;23(36):940821. PubMed PMID: 16361640.

26. Galderisi U, Jori FP, Giordano A. Cell cycle regulation and neural differentiation. Oncogene. 2003 Aug 11;22(33):5208-19. PubMed PMID: 12910258.

27. Vogelstein B, Lane D, Levine AJ. Surfing the p53 network. Nature. 2000 Nov 16;408(6810):30710. PubMed PMID: 11099028.

28. Thornberry NA, Lazebnik Y. Caspases: enemies within. Science. 1998 Aug 28;281(5381):1312-6. PubMed PMID: 9721091. Epub 1998/08/28. eng.

29. Yu C, Liu SL, Qi MH, Zou X, Wu J, Zhang J. Herbal medicine Guan Chang Fu Fang enhances 5fluorouracil cytotoxicity and affects drug-associated genes in human colorectal carcinoma cells. Oncology letters. 2015 Feb;9(2):701-8. PubMed PMID: 25621039. Pubmed Central PMCID: 4301488.

30. Brunelle JK, Letai A. Control of mitochondrial apoptosis by the Bcl-2 family. Journal of cell science. 2009 Feb 15;122(Pt 4):437-41. PubMed PMID: 19193868. Pubmed Central PMCID: 2714431.

31. Salakou S, Kardamakis D, Tsamandas AC, Zolota V, Apostolakis E, Tzelepi V, et al. Increased $\mathrm{Bax} / \mathrm{Bcl}-2$ ratio up-regulates caspase-3 and increases apoptosis in the thymus of patients with myasthenia gravis. In vivo. 2007 Jan-Feb;21(1):123-32. PubMed PMID: 17354625.

32. Danial NN. BCL-2 family proteins: critical checkpoints of apoptotic cell death. Clinical cancer research : an official journal of the American Association for Cancer Research. 2007 Dec 15;13(24):725463. PubMed PMID: 18094405.

33. Budram-Mahadeo V, Morris PJ, Latchman DS. The Brn-3a transcription factor inhibits the proapoptotic effect of p53 and enhances cell cycle arrest by differentially regulating the activity of the p53 target genes encoding Bax and p21(CIP1/Waf1). Oncogene. 2002 Sep 5;21(39):6123-31. PubMed PMID: 12203124. 


\section{Tables 1-2}

Tables 1-2 are not available with this version.

Figures 1-5

Figures 1-5 are not available with this version. 